This manuscript has been authored by UT-Battelle, LLC under Contract No. DE-AC05-00OR22725 with the U.S. Department of Energy. The United States Government retains and the publisher, by accepting the article for publication, acknowledges that the United States Government retains a non-exclusive, paidup, irrevocable, world-wide license to publish or re- produce the published form of this manuscript, or allow others to do so, for United States Government purposes. The Department of Energy will provide public access to these results of federally sponsored research in accordance with the DOE Public Access Plan(http://energy.gov/downloads/doepublic-access-plan).

\title{
Synergy of inelastic and elastic energy loss: Temperature effects and electronic stopping power dependence
}

\author{
E. Zarkadoula, ${ }^{1, *}$ Haizhou Xue, ${ }^{2}$ Yanwen Zhang, ${ }^{1,2}$ and William J. Weber ${ }^{2,1}$ \\ ${ }^{1}$ Materials Science $\&$ Technology Division, Oak Ridge National Laboratory, Oak Ridge, TN 37831, USA \\ ${ }^{2}$ Department of Materials Science $\&$ Engineering, \\ University of Tennessee, Knoxville, TN 37996, USA
}

\begin{abstract}
A combination of an inelastic thermal spike model suitable for insulators and molecular dynamics simulations is used to study the effects of temperature and electronic energy loss on ion track formation, size and morphology in $\mathrm{SrTiO}_{3}$ systems with pre-existing disorder. We find temperature dependence of the ion track size. We also find a threshold in the electronic energy loss for a given pre-existing defect concentration, which indicates a threshold in the synergy between the inelastic and elastic energy loss.
\end{abstract}

Keywords: radiation effects, molecular dynamics, ceramics, perovskite, defects

Electronic effects are of significant importance in a wide variety of fields where high energy irradiation processes take place, including nuclear applications, the semiconductor industry, material synthesis, modification and characterization $[1,2]$. The importance of the coupling of electronic and atomic processes in ionic and covalent materials has been emphasized in recent studies [1, 3-15], where it has been shown that these effects can have linearly additive [3-8] or competing [9-11] impacts on the defect production. A more recent study by Weber et al. [16] reveals a remarkable synergy between the inelastic energy loss and pre-existing damage, showing that the presence of pre-existing disorder in the system enhances the sensitivity of the material to the electronic energy loss effects. Furthermore, we previously showed [17] that the size of nanoscale ion tracks can be controlled by the concentration of pre-existing disorder in pre-damaged $\mathrm{SrTiO}_{3}$ systems. These results emphasize the importance of the pre-existing damage in the energy dissipation in the system and highlights the need to investigate further the role of the defects and defect excitation in microstructure alterations.

In the present paper, we study the effects of two factors on the synergy between electronic and atomic processes, and consequently in the ion track formation in pre-damaged $\mathrm{SrTiO}_{3}$, namely the effects of temperature and the effects of varying electronic energy loss.

We use a combination of an inelastic thermal spike (iTS) model suitable for insulators [18] and molecular dynamics (MD) simulations to model the energy dissipation due to irradiation into the system. The iTS model describes the energy exchange between the electronic and the atomic systems in terms of a set of two coupled heat diffusion equations, one for the electronic (1) and one for the atomic (2) system. The energy transfer from the electronic to the atomic lattice occurs via the electronphonon interactions.

We use a combination of an inelastic thermal spike (iTS) model suitable for insulators [18] and molecular dynamics (MD) simulations to model the energy dissipation due to irradiation into the system. The iTS model describes the energy exchange between the electronic and the atomic systems in terms of a set of two coupled heat diffusion equations, one for the electronic (1) and one for the atomic (2) system. The energy transfer from the electronic to the atomic lattice occurs via the electronphonon interactions.

$$
\begin{gathered}
C_{e} \frac{\partial T_{e}}{\partial t}=\frac{1}{r} \frac{\partial}{\partial r}\left[r K_{e} \frac{\partial T_{e}}{\partial r}\right]-g\left(T_{e}-T_{\alpha}\right)+A(r, t) \\
C_{a} \frac{\partial T_{a}}{\partial t}=\frac{1}{r} \frac{\partial}{\partial r}\left[r K_{a} \frac{\partial T_{a}}{\partial r}\right]+g\left(T_{e}-T_{a}\right)
\end{gathered}
$$

$\mathrm{C}_{e}$ and $\mathrm{C}_{a}$ are the specific heat coefficients of the electronic and atomic systems respectively, whereas $K_{e}$ and $K_{a}$ are the thermal conductivities of the electronic and the atomic system. The term $g$ is the electron-phonon coupling parameter, and $A(r, t)$ describes the energy deposition from the incident ion to the electrons [19]. The second term on the right side of the equations (1) and (2) represents the energy exchange between the electronic and atomic subsystems due to the difference between the electronic temperature $T_{e}$ and the atomic temperature $T_{a}$. 
The specific heat coefficients used for the electronic and atomic systems are $C_{e}=1 \mathrm{~J} \mathrm{~cm}^{-3} \mathrm{~K}^{-1}[18,20,21]$ and $C_{a}=0.544 \mathrm{~J} \mathrm{~g}^{-1} \mathrm{~K}^{-1}[22]$, respectively. To account for the decrease in the thermal conductivity due to the presence of the defects [23, 24], we used reduced values of $K_{e}$ and $K_{a}$ by an order of magnitude in comparison to the values for the perfect crystalline system. Such a decrease in the thermal conductivity is reasonably consistent with the large changes in thermal conductivity of $\mathrm{SrTiO}_{3}$ due to processing defects [25] or cation non-stoichiometry (a few percent) [26]. For the crystalline system $K_{e}$ is equal to $K_{e}=C_{e} D_{e}$ as previously suggested [18, 20,21], where $D_{e}$ is the thermal diffusivity, and $K_{a}$ is equal to 11.2 $\mathrm{W} \mathrm{m} \mathrm{m}^{-1} \mathrm{~K}^{-1}$ at $300 \mathrm{~K}$. Here we note that we do not take into account the difference in the thermal conductivity for the calculations at $500 \mathrm{~K}$ and $800 \mathrm{~K}$, as the difference is small and the effect in the energy deposition is negligible. The value of $g$ for a crystalline system is, as described elsewhere [18], $4.3 \times 10^{18} \mathrm{~W} \mathrm{~m}^{-3} \mathrm{~K}^{-1}$. We used a $35 \%$ larger value for the pre-damaged system to account for the decrease in the electron-phonon mean free path due to the defects.

The electronic energy loss due to $21 \mathrm{MeV} \mathrm{Ni}, 20 \mathrm{MeV}$ $\mathrm{Ti}, 12 \mathrm{MeV}$ and $21 \mathrm{MeV}$ Si ions irradiation are calculated using Stopping and Range of Ions in Matter (SRIM) code [27], and is determined as $9.74 \mathrm{keV} / \mathrm{nm}, 8.5 \mathrm{keV} / \mathrm{nm}, 6.9$ $\mathrm{keV} / \mathrm{nm}$, and $6.18 \mathrm{keV} / \mathrm{nm}$ respectively at about $120 \mathrm{~nm}$ irradiation depth. Figure 1 shows the energy deposition profile as a function of the distance from the ion path for these ions in pre-damaged $\mathrm{SrTiO}_{3}$ systems.

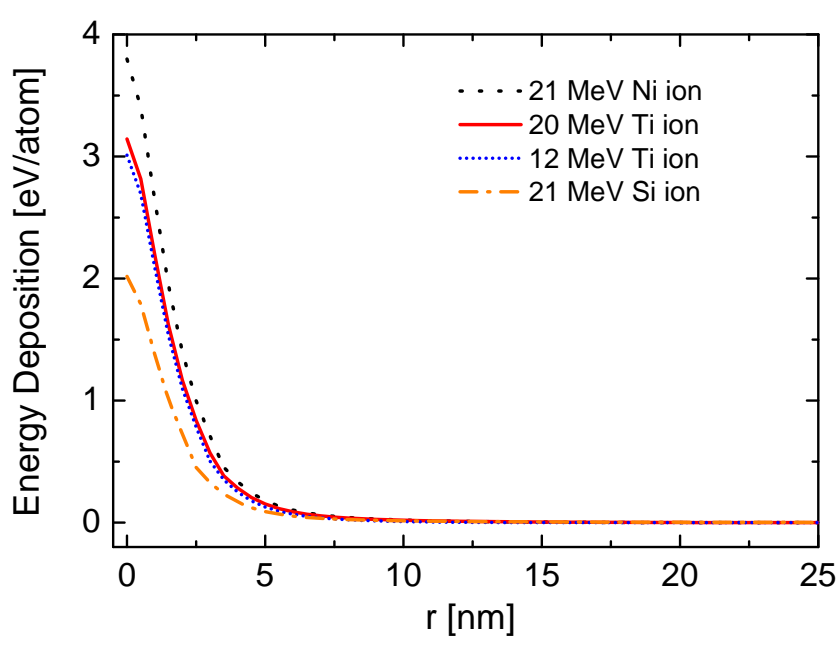

FIG. 1: Energy deposition profiles for $21 \mathrm{MeV} \mathrm{Ni}, 20 \mathrm{MeV}$ Ti, $12 \mathrm{MeV} \mathrm{Ti}$, and $21 \mathrm{MeV}$ Si ions in pre-damaged $\mathrm{SrTiO}_{3}$.

The energy profile calculated with the iTS model is used as input to the MD simulations, for which we use the DL_POLY [28] code. The pre-damaged systems were created by introducing Frenkel pairs (FP) randomly in the systems, which were consequently relaxed under constant pressure and temperature. The total number of atoms in each system is about 7 million. The irradiation of the systems is along the $\mathrm{z}$ direction under the constant-energy, constant-volume (NVE) ensemble at the target temperature, with a variable timestep, in a depth of about $26 \mathrm{~nm}$. The atoms contained in the $\mathrm{x}$ and $\mathrm{y}$ boundary of the MD box, in a layer of about $1 \mathrm{~nm}$ thickness, are connected to a Langevin thermostat at $300 \mathrm{~K}$ to emulate the effect of energy dissipation into the sample. The defects are identified using the sphere criterion [29], with a cut-off radius of $0.75 \AA$. We use empirical potentials by McCoy et al. [30] joined to the ZBL [31] repulsive potentials for short distances. The ZBL potential was used for all pair interactions.

(a)

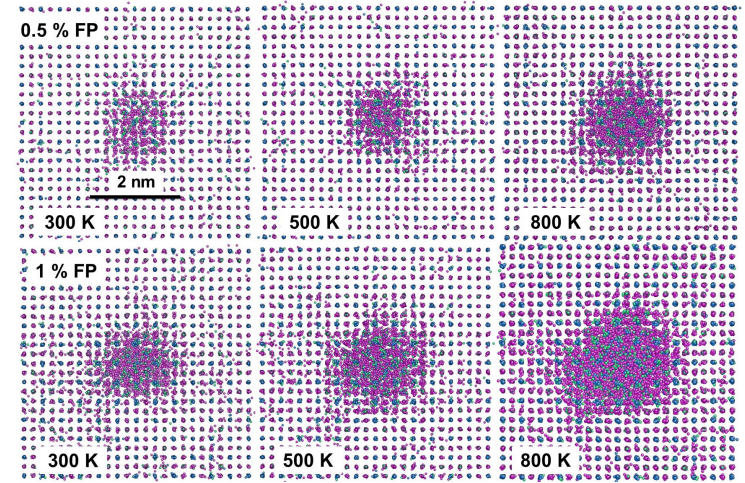

(b)

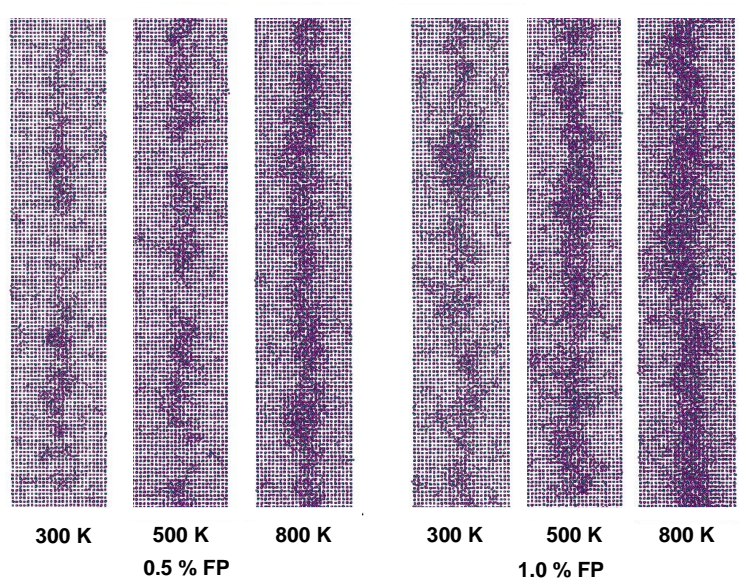

FIG. 2: (a) Cross sections of systems containing $0.5 \%$ FP (top) and $1.0 \%$ (bottom) along the [001] direction after irradiation with $21 \mathrm{MeV} \mathrm{Ni}$ at $300 \mathrm{~K}, 500 \mathrm{~K}$ and $800 \mathrm{~K}$. (b) Slices along the ion path of systems containing $0.5 \%$ (left) and $1.0 \%$ (right) FP after irradiation with $21 \mathrm{MeV} \mathrm{Ni}$ at 300 $\mathrm{K}, 500 \mathrm{~K}$ and $800 \mathrm{~K}$. Sr is shown in blue, Ti in green, and $\mathrm{O}$ in purple.

To study the effects of temperature on the synergy of the elastic and inelastic energy loss, we used two systems containing about $0.5 \% \mathrm{FP}$ and $1 \% \mathrm{FP}$, where we applied the energy deposition that corresponds to $21 \mathrm{MeV}$ Ni ions at $300 \mathrm{~K}, 500 \mathrm{~K}$ and $800 \mathrm{~K}$. To study the effects of the 
coupling of different electronic energy loss and the preexisting disorder we used two systems containing about $2 \%$ and $12 \% \mathrm{FP}$, where we applied the energy profiles shown in Fig. 1 at $300 \mathrm{~K}$ simulation temperature.

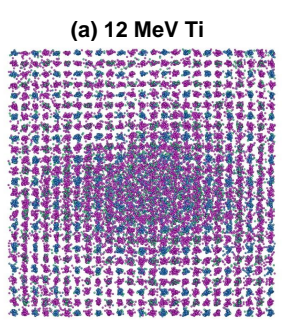

$12 \%$ FP (b) $20 \mathrm{MeV} \mathrm{Ti}$

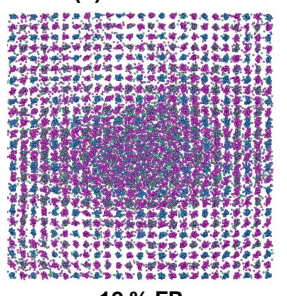

$12 \%$ FP (c) $21 \mathrm{MeV} \mathrm{Ni}$

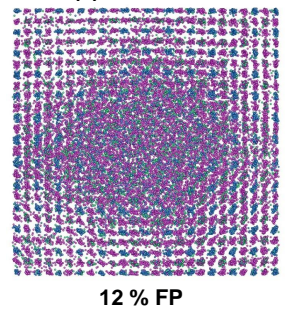

FIG. 3: Cross sections of the systems containing $12 \% \mathrm{FP}$ along the [001] direction after irradiation with (a) $12 \mathrm{MeV}$ Ti, (b) $20 \mathrm{MeV} \mathrm{Ti}$ and (c) $21 \mathrm{MeV} \mathrm{Ni}$ at $300 \mathrm{~K}$. The area shown is $5 \mathrm{~nm} \times 5 \mathrm{~nm}$. Sr is shown in blue, Ti in green, and $\mathrm{O}$ in purple.

Figure 2 (a) shows the cross sections of systems containing $0.5 \%$ and $1 \% \mathrm{FP}$ at the end of the simulation time at different temperatures. We see that the ion track diameter increases for higher temperature. Figure 2 (b) illustrates perpendicular slices (parallel to $\mathrm{z}$ axis) of the same systems, where we see that for a given concentration of pre-existing defects the resulting ion tracks become more continuous.

Higher temperature means that the energy needed to cause melting of the material is less, therefore the damage efficiency is higher as the temperature increases, and the resulting ion tracks are larger for higher temperatures. Such behavior has been observed in experiments in pyrochlore for $40 \mathrm{keV} / \mathrm{nm}$ energy loss at $8 \mathrm{~K}$ and $300 \mathrm{~K}$ [32] and in bismuth for energy loss about $30-38 \mathrm{keV} / \mathrm{nm}$ for a range of temperatures from $20 \mathrm{~K}$ to $300 \mathrm{~K}$ [33]. The non-continuous morphology of the ion tracks for low disorder level systems, as well as differences in the track diameter size, in different depth for larger disorder level systems, as discussed elsewhere [17], can be attributed to the inhomogeneity of the defect distribution along the ion path.

Irradiation of systems with $2 \%$ pre-existing $\mathrm{FP}$ with $9.74 \mathrm{keV} / \mathrm{nm}, 8.5 \mathrm{keV} / \mathrm{nm}$ and $6.9 \mathrm{keV} / \mathrm{nm}$ electronic stopping power results in the formation of an ion track only for $9.74 \mathrm{keV} / \mathrm{nm}$ electronic energy loss. For the lower values of stopping power, the damage created during the irradiation has recovered, with only a few point defects and point defect clusters surviving after the system relaxation. However, irradiation of systems that contains $12 \% \mathrm{FP}$ results in formation of ion tracks in all three cases of electronic stopping power. For higher electronic energy loss the ion track is larger, more homogeneous and more continuous. Figure 3 shows the cross sections of the higher defect density (12\% FP) systems, where the ion track diameter ranges from about $1.8 \mathrm{~nm}$

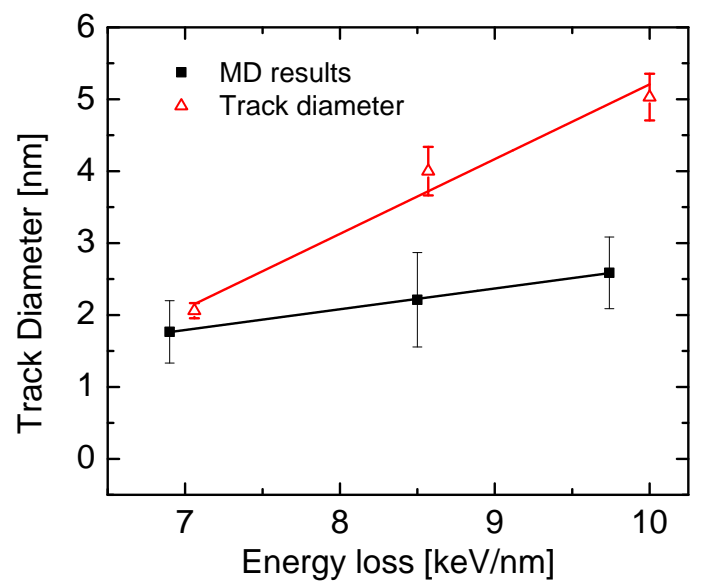

FIG. 4: Ion track diameter as a function of the electronic energy loss in higher pre-existing disorder systems (12\% FP initial damage for the MD results, $11 \%$ disorder for the experimental data). The solid black squares represent MD results. The open red triangles represent experimental data. The error bars represent the standard deviation over different measurements of the diameter along the ion tracks. The fit for both data sets is plotted (black line for the MD results, red line for the experimental data) and indicates a linear relationship between the track diameter and the electronic stopping power.

$\pm 0.4 \mathrm{~nm}$ to $2.6 \mathrm{~nm} \pm 0.5 \mathrm{~nm}$ for energy loss from 6.9 to $9.74 \mathrm{keV} / \mathrm{nm}$ respectively. The boundaries of these ion tracks are difficult to distinguish due to the higher level of disorder in the surrounding material. The ion track diameter through the samples that contain $12 \%$ FP initial damage as a function of the electronic energy loss is shown in Figure 4, along with experimental data for samples with $11 \%$ initial disorder. The similar trends seen here suggest a good agreement between the computational and experimental results, and both indicate a linear relationship between average track diameter and the electronic stopping power. Irradiation with $21 \mathrm{MeV}$ Si ions $(6.18 \mathrm{keV} / \mathrm{nm}$ electronic energy loss) did not produce ion tracks in either system.

The presence of the pre-existing damage means that less energy is needed in order for the defects to start percolating. With increasing electronic energy loss, more energy is deposited to the lattice, including the pre-existing defects, and the ion tracks become more continuous and larger in size. The fact that no ion track is produced for $6.18 \mathrm{keV} / \mathrm{nm}$ energy loss indicates that despite the presence of the defects, the energy deposited is not sufficient to enhance the disorder in the vicinity of the ion path and create a track. Therefore these results suggest that there is a threshold for the synergy of the inelastic energy loss and the atomic defects, which depends on the combination of the pre-existing disorder and the stopping 
power.

In summary, our findings show that there is a temperature dependence of the ion track size and morphology in pre-damaged systems of $\mathrm{SrTiO}_{3}$; higher irradiation temperature results in larger and more homogeneous ion tracks. Additionally, we found that a given concentration of pre-existing defects enhances the sensitivity of $\mathrm{SrTiO}_{3}$ to the energy loss up to a threshold value for synergy of elastic and inelastic energy loss to occur. This indicates that the synergy between the electronic and atomic processes depends on the combination of the pre-existing damage level and electronic stopping power, meaning that the deposited energy needs to be sufficient to enhance the disorder and result in ion track formation.

The results discussed here demonstrate the importance of the interaction of the pre-existing disorder with the electronic effects during irradiation and in the damage evolution. Further investigation of the coupled effects of inelastic energy loss and pre-existing defects in more materials is needed in order to better understand the processes that take place during irradiation and predict the materials' performance in extreme conditions.

This work was supported by the U.S. Department of Energy, Office of Science, Basic Energy Sciences, Materials Sciences and Engineering Division. This research used resources of the National Energy Research Scientific Computing Center, supported by the Office of Science, US Department of Energy under Contract No.DEAC02$05 \mathrm{CH} 11231$.

* Electronic address: zarkadoulae@ornl.gov

[1] W. J. Weber, D. M. Duffy, L. Thomé, Y. Zhang, Curr. Opin. Solid State Mater. Sci. 19 (2015) 1-11.

[2] Y. Zhang, A. Debelle, A. Boulle, P. Kluth, F. Tuomisto, Curr. Opin. Solid State Mater. Sci. 19 (2015) 19-28.

[3] M. Toulemonde, W. j. Weber, G. Li, V. Shutthanandan, P. Kluth, T. Yang, Y. Wang, Y. Zhang, Phys. Rev. B 83 (2011) 054106.

[4] L. Thomé, A. Debelle, F. Garrido, P. Trocellier, Y. Serruys, G. Velisa, S. Miro, Appl. Phys. Lett. 102 (2013) 141906.

[5] M. Sall, I. Monnet, C. Grygiel, B. Ban dEtat, H. Lebius, S. Leclerc, E. Balanzat, EPL 102 (2013) 26002.

[6] Y. Zhang, D. S. Aidhy, T. Varga, S. Moll, P. D. Edmondson, F. Namavar, K. Jin, C. N. Ostrouchov, W. J. Weber, Phys. Chem. Chem. Phys. 16 (2014) 8051-8059.

[7] Y. Y. Wang, C. Grygiel, C. Dufour, J. R. Sun, Z. G. Wang, Y. T. Zhao, G. Q. Xiao, R. Cheng, X. M. Zhou, J. R. Ren, S. D. Liu, Y. Lei, Y. B. Sun, R. Ritter, E. Gruber, A. Cassimi, I. Monnet, S. Bouffard, F. Aumayr, M. Toulemonde, Sci. Rep. 4 (2014) 5742.

[8] M. Backman, F. Djurabekova, O. H. Pakarinen, K. Nordlund, Y. Zhang, M. Toulemonde, W. J. Weber J. Phys. D: Appl. Phys. 45 (2012) 505305.

[9] A. Debelle, M. Backman, L. Thomé, W. J. Weber, M. Toulemonde, S. Mylonas, A. Boulle, O. H. Pakarinen,
N. Juslin,F. Djurabekova, K. Nordlund, F. Garrido, D. Chaussende Phys. Rev. B 86 (2012) 100102.

[10] W. J. Weber, Y. Zhang, H. Y. Xiao, L. M. Wang, RSC Advances 2 (2012) 595-604.

[11] Y. Zhang, T. Varga, M. Ishimaru, P.D. Edmondson, H. Xue, P. Liu, S. Moll, F. Namavar, C. Hardiman, S. Shannon, W.J. Weber Nucl. Instrum. Methods. Phys. Res. B 327 (2014) 33-43.

[12] S. J. Zinkle, V. Skuratov, D. T. Hoelzer, Nucl. Instrum. Methods Phys. Res. B 191 (2002) 758-766.

[13] C. Kinoshita, K. Yasuda, S. Matsumura, M. Shimada, Metall. Mater. Trans. A 35 (2004) 2257-2266.

[14] A. Kamarou, W. Wesch, E. Wendler, Phys. Rev. B 78 (2008) 054111.

[15] D. M. Duffy, S. L. Daraszewicz, J. Mulroue, Nucl. Instrum. Methods Phys. Res. B 277 (2012) 21-27.

[16] W. J. Weber, E. Zarkadoula, O. H. Pakarinen, R. Sachan, M. F. Chisholm, P. Liu, H. Xue, K. Jin, Y. Zhang, Sci. Rep. 5 (2015) 7726.

[17] E. Zarkadoula, O. H. Pakarinen, H. Xue, Y. Zhang, W. J. Weber, submitted for publication

[18] M. Toulemonde, W. Assman, C. Dufour, A. Meftah, F. Studer, C. Trautmann, Mat. Fys. Medd. K. Dan. Vidensk. Selsk. 52 (2006) 263-292.

[19] M. P. R. Waligorski, R. N. Hamm, R. Katz, Nucl. Tracks Radiat. Meas. 11 (1986) 309-319.

[20] M. Toulemonde, J. M. Costantini, C. Dufour, A. Meftah, E. Paumier, F. Studer, Nucl. Instr. Meth B 116 (1996) 37.

[21] A. Meftah, J. M. Costantini, N. Khalfaoui, S. Boudjadar, J. P. Stoquert, F. Studer, M. Toulemonde, Nucl. Instrum. Methods Phys. Res. B 237 (2005) 563-574.

[22] http://www.toplent.com/SrTiO3.htm

[23] L. L. Snead, S. J. Zinkle, D. P. White, J. Nucl. Mater. 340 (2005) 187-202.

[24] P. B. Weisensee, J. P. Feser ,D. G. Cahill, J. Nucl. Mater. 443 (2013) 212-217.

[25] D-W. Oh, J. Ravichandran, C-W. Liang, W. Siemons, B. Jalan, C. M. Brooks, M. Huijben, D. G. Schlom, S. Stemmer, L. W. Martin, A. Majumdar, R. Ramesh, D. G. Cahill, Appl. Phys. Lett. 98 (2011) 221904.

[26] E. Breckenfeld, R. Wilson, J. Karthik, A. R. Damodaran, D. G. Cahill, L. W. Martin, Chem. Mater. 24 (2012) 331337.

[27] J. F. Ziegler, J. P. Biersack, M. D. Ziegler, SRIM The Stopping and Range of Ions in Matter. (SRIM Co., Chester, MD, USA, 2008).

[28] I. T. Todorov, B. Smith, M. T. Dove, K. Trachenko, J. Mater. Chem. 16 (2006) 1911.

[29] I.T. Todorov, W. Smith, THE DL POLY 4 USER MANUAL, v.4 2012, available at http://www.afs.enea.it/software/dlpoly/USRMAN4.03.pdf

[30] M. A. McCoy, R. W. Grimes, W. E. Lee, Philos. Mag. A 75 (1997) 833-846.

[31] J. F. Ziegler, J. P. Biersack, U. Littmark, The Stopping and Range of Ions in Matter (Pergamon, New York, 1985).

[32] J. Zhang, M. Lang, R. C. Ewing, R. Devanathan, W. J. Weber, M. Toulemonde, J. Mater. Res. 25 (2010) 13441351.

[33] Ch. Dufour, F. Beuneu, E. Paumier, M. Toulemonde, Europhys. Lett., 45 (1999) 585-590. 